\title{
Tuning of MST Neurons to Spiral Motions
}

\author{
Michael S. A. Graziano, Richard A. Andersen, and Robert J. Snowdena \\ Department of Brain and Cognitive Sciences, Massachusetts Institute of Technology, Cambridge, Massachusetts 02139
}

Cells in the dorsal division of the medial superior temporal area (MSTd) have large receptive fields and respond to expansion/contraction, rotation, and translation motions. These same motions are generated as we move through the environment, leading investigators to suggest that area MSTd analyzes the optical flow. One influential idea suggests that navigation is achieved by decomposing the optical flow into the separate and discrete channels mentioned above, that is, expansion/contraction, rotation, and translation. We directly tested whether MSTd neurons perform such a decomposition by examining whether there are cells that are preferentially tuned to intermediate spiral motions, which combine both expansion/contraction and rotation components. The finding that many cells in MSTd are preferentially selective for spiral motions indicates that this simple three-channel decomposition hypothesis for MSTd does not appear to be correct. Instead, there is a continuum of patterns to which MSTd cells are selective.

In addition, we find that MSTd cells maintain their selectivity when stimuli are moved to different locations in their large receptive fields. This position invariance indicates that MSTd cells selective for expansion cannot give precise information about the retinal location of the focus of expansion. Thus, individual MSTd neurons cannot code, in a precise fashion, the direction of heading by using the location of the focus of expansion. The only way this navigational information could be accurately derived from MSTd is through the use of a coarse, population encoding. Positional invariance and selectivity for a wide array of stimuli suggest that MSTd neurons encode patterns of motion per se, regardless of whether these motions are generated by moving objects or by motion induced by observer locomotion.

[Key words: visual system, motion perception, visual pathways, extrastriate cortex, area $M S T$, parietal cortex]

The medial superior temporal area in the macaque visual system contains at least two major subdivisions: a ventral-lateral one (MSTl), and a dorsal one (MSTd) (Desimone and Ungerleider,

\footnotetext{
Received Jan. 5, 1993; revised June 3, 1993; accepted June 23, 1993.

We thank Stefan Treue and Gail Robertson for their help in all phases of the experiment, Bard Geesaman for his help in the recording experiments, and John Rosen for his advice on statistics. This work was supported by NIH Grant EY07492, ONR Contract N00014-89-J1236, and a grant from the McDonnell-Pew Program in Cognitive Neuroscience.

Correspondence should he addressed to Richard A. Andersen, Department of Brain and Cognitive Sciences, Massachusetts Institute of Technology, 77 Massachusetts Avenue (E25-236), Cambridge, MA 02139.

a Present address: Vision Research Unit, School of Psychology, University of Wales College of Cardiff, Cardiff CF1 3 YG, UK.

Copyright (C) 1994 Society for Neuroscience $0270-6474 / 94 / 140054-14 \$ 05.00 / 0$
}

1986; Saito et al., 1986; Ungerleider and Desimone, 1986a,b; Komatsu and Wurtz, 1988a,b). Cells in the ventral portion are directionally selective and respond to translating bars or dots, much like cells in MT or V1 (Hubel and Wiesel, 1962; Maunsell and Van Essen, 1983a,b; Albright, 1984; Livingstone and Hubel, 1988). Cells in the dorsal portion have much larger receptive fields and respond to large field stimuli. While some MSTd neurons respond to simple translational motion, many others respond to clockwise rotation, counterclockwise rotation, expansion, or contraction (Sakata et al., 1985, 1986; Saito et al., 1986; Tanaka et al., 1986, 1989; Tanaka and Saito, 1989). Since these motions are generated by moving through the environment, it has been suggested by several groups that area MSTd may play a role in the analysis of optical flow (Saito et al., 1986; Roy and Wurtz, 1990). In this article we first describe a new class of stimuli that we have developed, which enable us to present a wide range of motion patterns that are identical in every way except for their global structure. We then describe how these stimuli were used to test whether MSTd neurons are selective only for expansion, rotation, and translation motions, or if they are tuned to a continuous array of motion patterns. Finally, we examine whether cells maintain their complex motion selectivity at different positions in their receptive fields. These results have important implications for the possible role of MSTd in visual navigation by flow field analysis.

Early studies of MST (Sakata et al., 1985, 1986; Saito et al., 1986; Tanaka et al., 1986, 1989; Tanaka and Saito, 1989) used hand-held stimuli and optically projected stimuli that contained many variables other than the global pattern of motion. For instance, the texture elements in the expansion/contraction stimuli changed size and density, and, when a zoom lens was used, the luminance of the stimulus varied. The individual pixels had curved trajectories for the rotation stimuli and accelerations or decelerations for the expansion and contraction stimuli. Tanaka and Saito and collcagucs were aware of these potential unwanted variables and performed control experiments in which they showed that manipulation of the individual variables had no substantial effect on the selectivity of MSTd neurons. However, no method was available to standardize and equate the different stimuli so that they could be directly compared. In our experiments we used computer-generated stimuli that eliminated all of these unwanted artifacts. The stimuli were identical in every way except for the global pattern of motion. Therefore, as described below, we were able to study the properties of MSTd cells by changing one variable without generating unwanted changes in other variables.

Recently Duffy and Wurtz (1991a) made the important finding that MSTd neurons respond not only to expansion/contraction, rotation, or translation, but often to two or all three of these motions. Their discovery of double- and triple-com- 
ponent cells suggested to us that MSTd neurons may be tuned to intermediate stimuli. For instance, a cell that responds to clockwise rotation and to expansion may be even more responsive to an intermediate, clockwise-expanding spiral motion. We designed an experiment to test this hypothesis. We tested cells with expansions, contractions, rotations, and also with spiraling stimuli. All of these stimuli were identical except for their global pattern of motion. We plotted the results in a coordinate system that contained two orthogonal axes, one representing expansion/ contraction, and the other representing clockwise/counterclockwise rotation. The intermediate directions represented different types of spiral stimuli. If MSTd neurons were selective only for expansion/contraction and rotation, then the best directions of all the neurons should line up along the cardinal axes of this space. If MSTd cells were selective for a range of motion patterns, then the best directions should be more widely distributed, and include different spiral stimuli as well.

The idea of basis vectors is of course not uncommon in vision. Although we see a wide range of colors, they are derived from the relative activities of only three channels corresponding to the three cone classes. In a similar vein, we would need only vertically and horizontally tuned direction-selective neurons in areas VI and MT, and diagonal motions could be encoded by the relative activities of these two channels. Of course we know that there is a continuous array of best directions in V1 and MT, and not two orthogonal motion channels. It is also plausible that complex motion would be encoded in MSTd by three orthogonal motion channels, corresponding to expansion/contraction, rotation, and translation. Alternatively, like $\mathrm{V} 1$ and MT, MSTd might encode motion with a continuous array of best directions. Whether MSTd contains only a small number of motion channels, or a continuous distribution of best directions, was the central question of our experiment.

The idea of basis vectors has a direct bearing on how optical flow may be analyzed. Optical flow can be decomposed into expansion/contraction motions generated by movement of the observer through the environment, translations produced by rotations of the eyes, and rotations generated by head movements and eye torsions (Longuet-Higgins and Prazdny, 1980; Koenderink and van Doorn, 1981). When the obscrver moves, the focus of expansion is also the direction of heading and can be used for navigation (Gibson, 1950; Warren and Hannon, 1988). If the eyes move as well, for example, to track an object on the ground, then a translation component is added to the expansion and the new focus of the composite flow is at the point of fixation (Longuet-Higgins and Prazdny, 1980; Koenderink and van Doorn, 1981; Regan and Beverly, 1982; Warren and Hannon, 1988). The true focus of expansion could be recovered by decomposing the flow field into its various components, and since humans can accurately determine the true direction of heading under such conditions, it seems likely that some form of decomposition is performed (Rieger and Lawton, 1985; Warren and Hannon, 1988; Heeger and Jepson, 1990). A vector basis encoding in MSTd would conform quite naturally to such a decomposition (Longuet-Higgins and Prazdny, 1980; Kocnderink and van Doorn, 1982; Orban et al., 1992). However, if MSTd does not decompose the optical flow field into basis vectors, then it must not be contributing to navigation by this particular method.

In another experiment, we examined position invariance, that is, a cell's consistent selectivity to a stimulus regardless of where the stimulus falls in the cell's receptive field. This test also has important implications for visual navigation. If MSTd cells are position invariant, then individual cells will not give precise information about the focus of expansion, and therefore will not provide unambiguous information about the direction of heading. Such a finding would argue that only populations of broadly tuned MSTd cells could provide this information; that is, a coarse coding mechanism would be required if MSTd is involved in optical flow analysis. In the most general formulation, MSTd may analyze complex motion per se, regardless of whether it is generated by the motion of individual objects in the environment or by the motion of the observer through the environment.

Some of these results have already been published in abstract form (Andersen et al., 1990; Graziano et al., 1990).

\section{Materials and Methods}

\section{Animal preparation}

Responses from MST cells were studied from two hemispheres of two awake rhesus monkeys performing a fixation task. $\Lambda$ scleral search coil and an acrylic skull cap were implanted under general anesthesia and sterile surgical conditions 1 week before training began. During training the monkey was water deprived and rewarded for correct responses with apple juice. Once training was complete, a second surgery was performed in which a recording chamber was added to the skull cap. Electrode penetrations were made through the oil-filled recording chamber. The chamber for the first monkey was positioned vertically for a dorsal approach to MST. The chamber for the second monkey was positioned over striate cortex for a diagonal posterior approach. Recording sessions lasted $4-8 \mathrm{hr}, 5 \mathrm{~d}$ a week, and typically three to five cells were studied in detail per day. The animals were given ample rest periods during each recording session.

\section{Fixation task and data collection}

Each trial was initiated by the illumination of a light-emitting diode (I.FD) placed centrally in the monkey's field of view, $57 \mathrm{~cm}$ away. The monkey was required to pull a lever and fixate the LED within a time window of 150-600 msec after its onset. After 2-3 sec the LED was dimmed, and the monkey was required to release the lever within a time window of 150-600 msec after the dimming, to receive an apple juice reward. If during the trial the animal's eye moved at a speed above $15 \% \mathrm{sec}$, as in a saccade away from the fixation point, then the trial was terminated without reward. Eye position was monitored every $35 \mathrm{msec}$ and the standard deviation of eye position on successful trials was less than $y \mathrm{~min}$ of arc for both animals. During the period of fixation, a visual stimulus was displayed on a large, $25 \mathrm{~cm} \times 30 \mathrm{~cm}$ cathode ray tube (phosphor 31 ) placed $57 \mathrm{~cm}$ in front of the monkey. Data collection, events for the fixation task, and stimulus presentation were controlled by a computer.

\section{Stimuli}

Cells were tested with five standard sets of stimuli. Within each set the stimuli were presented in a pseudorandom interleaved fashion, one stimulus per trial, and the cell's baseline activity was measured by interleaved trials in which no stimulus was presented. Each stimulus had a duration of 2 sec.

Basic selectivity. The first set tested basic stimulus selectivity by using eight interleaved stimuli: expansion, contraction, clockwise rotation, counterclockwise rotation, and the four cardinal directions of translation (up, down, right, and left). Each stimulus was generated off line and stored as a sequence of still frames. A single movie was used for each type of stimulus, and as a result the position of the dots was the same across trials within a given stimulus class. Control experiments showed that the tuning of MST neurons is not sensitive to the exact pattern of the dots, and is even invariant for the features (e.g., dots, objects, illusory contours) used to compare the motion patterns (Geesaman and Andersen, 1992). During testing, the movies were presented at a frame rate of $35 \mathrm{~Hz}$. The stimuli were composed of 126 dots displayed within a circular window $20^{\circ}$ in diameter. Each dot moved for a $500 \mathrm{msec}$ lifetime, and then was assigned a new random location within the circle, and given a trajectory and speed appropriate to its new location. The 

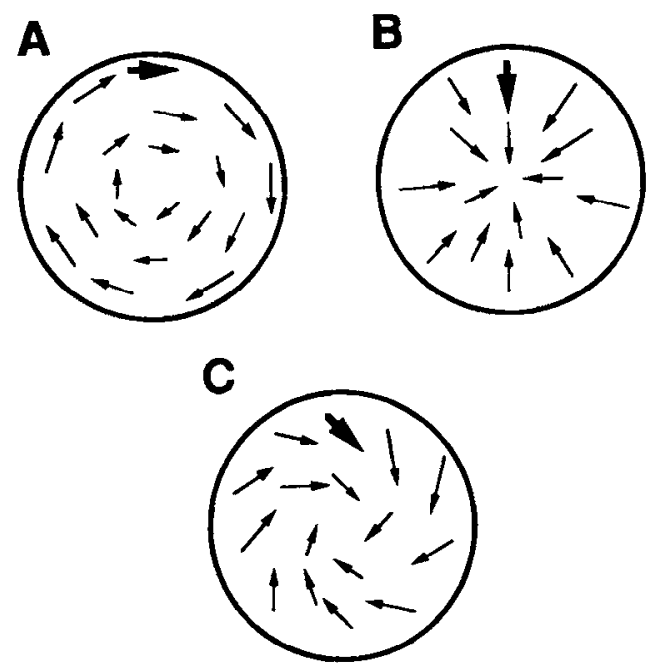

Figure 1. Schematic drawing of random dot stimuli. Each arrow represents the trajectory of a single dot. Arrow length indicates the speed. As shown by the thick arrow, the speed was the same for rotation $(A)$, contraction $(B)$, and spiral $(C)$; only the direction of motion varied.

dots were relocated asynchronously, to avoid a coherent flickering of the stimulus. If a moving dot traveled outside the window of the display, it was immediately relocated to a new, random location within the window. This constant reshuffling virtually eliminates pattern artifacts because the pattern of the dots was constantly, randomly changing. The reshuffling also eliminates density artifacts, since each local region in the display has approximately the same number of points at any time. As a result the mean luminances are also flat across the display. During each lifetime, a dot moved in a straight line with constant speed, thereby eliminating curvature artifacts for rotation, and acceleration artifacts for expansion and contraction. Finally, the expansions, contractions, and rotations had identical speed distributions. The speed of a dot located at distance $r$ from the center of the stimulus was given by the formula $S=0.3 \times r$. This value corresponds to a rotational speed of 0.3 radians/sec in the case of rotation. Figure $1, A$ and $B$, shows how a dot near the top edge moves to the right in clockwise rotation, and downward in contraction. The speed of the dot is the same in both stimuli; only the direction has changed. The translational motion stimuli moved at $4.4^{\circ}$ of visual angle per second, equal to the average dot speed in the expanding, contracting, and rotating stimuli. Because these eight stimuli were equated for density, size, and speed, and the trajectories of the dots contained no acceleration or curvature, the response of a cell could be attributed to only one variable: the global motion of the stimulus.

Spiral motion. The second stimulus set contained eight stimuli: expansion, contraction, clockwise rotation, counterclockwise rotation, and the four intermediate directions of spiral motion (expanding clockwise spiral, expanding counterclockwise spiral, contracting clockwise spiral, and contracting counterclockwise spiral). These stimuli were used to generate tuning curves in a "spiral space" in which expansion, contraction, clockwise rotation, and counterclockwise rotation are the four cardinal directions (see Fig. $7 A$ ). The stimuli were matched for size $\left(20^{\circ}\right.$ diameter), pattern, and density, and had no curvature or acceleration. They were also matched for speed. Figure 1 shows how a dot near the top edge had the same speed in clockwise rotation, contraction, and the contracting clockwise spiral; only the direction of the vector was changed, thereby changing the pitch of the spiral motion. Thus, a stimulus-selective response of a cell could be attributed to only one variable, the pitch of the spiral motion.

Position invariance. The third stimulus set used $10^{\circ}$ diameter stimuli at five different retinal positions (see Fig. 10A). These positions lay in an overlapping cloverleaf arrangement, covering $20^{\circ}$ of visual angle. In the regions of overlap, the direction of motion reversed depending on stimulus position. Therefore, if a cell responded in the same way to all five positions, the response could not be explained by local patches of directional selectivity. The cell must be selective for the global pattern of motion.

Rotation cells were tested with both directions of rotation at each of the five locations, forming an interleaved set of 10 stimuli. Expansion/ contraction cells were tested with expansion and contraction at each of the five locations, again forming an interleaved set of 10 stimuli.

Spiral position invariance. The fourth stimulus set was used to generate spiral tuning curves at two positions in the receptive field: an upper and a lower position. The expansion, contraction, rotation, and spiral stimuli used in this test were the same as those described above for spiral motion, except that the size was $16.5^{\circ}$ across. The two overlapping positions therefore covered $25^{\circ}$ of visual angle.

Size. In the fifth stimulus set, $10^{\circ}$ and $20^{\circ}$ diameter stimuli were presented in an interleaved fashion to test the dependence of responses on stimulus size.

\section{Histology}

In the last week of recording from monkey 1 , lesions were made by passing a small direct current through the recording electrode. The animal was killed with sodium pentobarbital and perfused transcardially with heparinized saline and then formalin. Guide wires were lowered into the brain at selected chamber coordinates and used as landmarks for blocking the posterior parietal cortex. Coronal sections were made at $30 \mu \mathrm{m}$ thickness and stained alternately with thionin for cytoarchitecture and with the Gallyas method (Gallyas, 1979) for myeloarchitecture.

Damage from electrode penetrations and lesions corresponded well with the location of MST as described by previous researchers (Desimone and Ungerleider, 1986; Komatsu and Wurtz, 1988a). Successful penetrations that had yielded expansion, contraction, and rotation responses were clustered in the upper bank of the superior temporal sulcus, dorsal to area MT. Area MT was identified by its characteristic dense myelin staining. The recording sites in MST were concentrated in another densely myelinated zone that is coextensive with much of MSTd (Desimone and Ungerleider, 1986).

\section{Results}

One hundred sixty-nine cells were studied in two hemispheres of two rhesus monkeys. Neurons were studied using the five different tests outlined in Materials and Methods.

\section{Basic selectivity}

Ninety-five MST cells were tested for their basic stimulus selectivity. They were tested with expansion, contraction, two directions of rotation, and four directions of translational motion, equated for pattern, size, speed, and density, and without curvature or acceleration. Therefore, any selective response can be attributed to the global pattern of motion of the stimulus, not to an incidental artifact.

Seventy-five cells (79\%) responded significantly above baseline to at least one stimulus ( $t$ test, $p<0.05$ ). Figure 2 shows rasters and histograms for a cell that preferred expansion. This cell is typical in its strong and sustained response to the stimulus. There is little response to the other stimuli. Figure 3 shows a ccll that preferred counterclockwise rotation. Again, there is little response to the other stimuli. However, other cells responded to two or even three types of motion. Figure 4 shows a cell that responded both to expansion and to counterclockwise rotation, and Figure 5 shows a cell that responded to contraction, clockwise rotation, downward motion, and leftward motion.

Figure $6 A$ shows the distribution of responses. The triangular space depicted in this graph shows the relative contributions of each of the three types of motion. For example, a cell plotted near the expansion/contraction vertex responded best to expansion or contraction, a cell plotted near the clockwise/counterclockwise vertex responded best to rotation, a cell plotted near the up/down/right/left vertex responded best to one of the translational motion stimuli, and a cell plotted near the center responded equally well to all three types of stimuli (see caption for details). This graph gives no information about absolute 

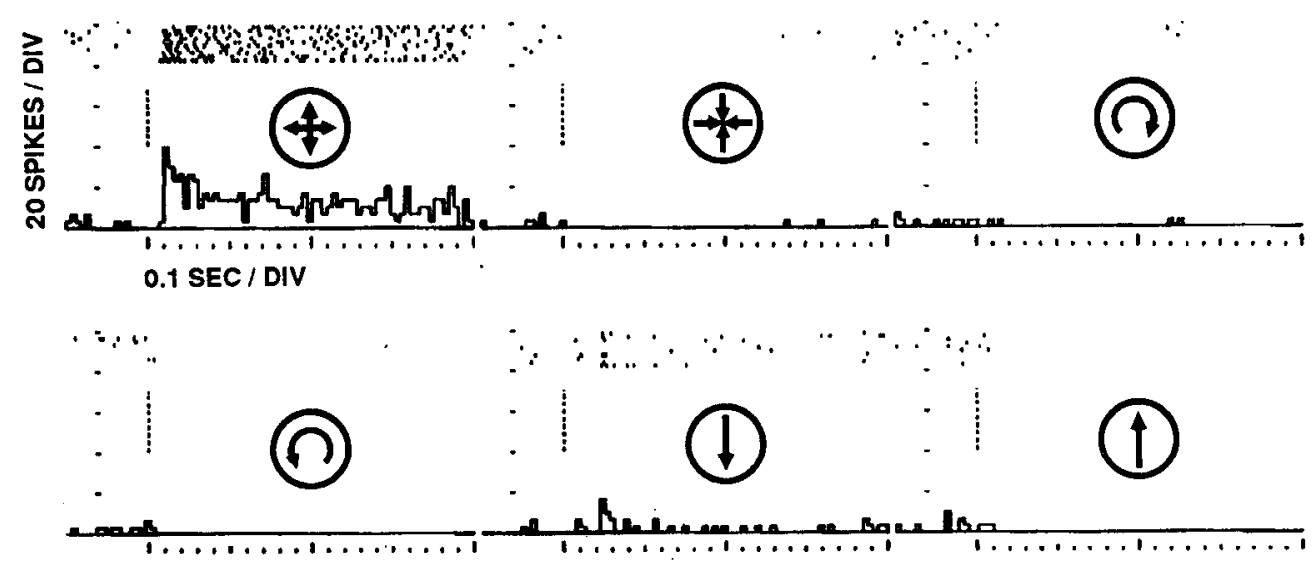

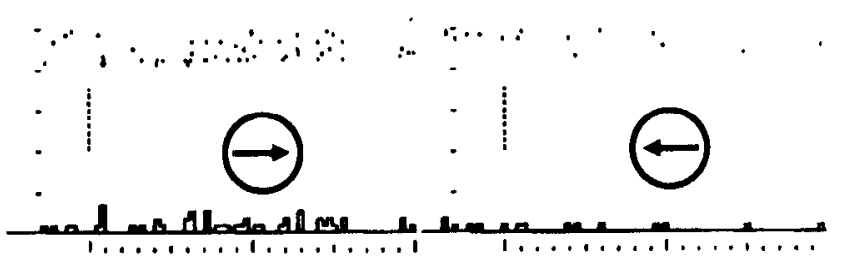

response strength. Instead, it is intended to show the relative response strength to each of the three motion categories. All the cells included in this graph responded significantly above baseline to at least one stimulus ( $t$ tcst, $p<0.05$ ). There is clearly a broad range of response types. Every combination of stimulus selectivity is represented, and there is no clustering into expansion cells, rotation cells, or translation cells. Many cells fell near the center of the graph, indicating that they responded to all three types of stimuli.

This distribution of responses may be biased because only two directions are tested for rotation (clockwise, counterclockwise) and for expansion/contraction, whereas four directions are tested for translation. As was pointed out in Materials and Meth- ods, rotation and expansion/contraction can be considered four directions in a continuous motion space just as up, down, right, and left can be considered four directions in a continuous translation space. Figure $6 B$ illustrates how the distribution can change by plotting a more fair comparison between expansion/contraction, clockwise/counterclockwise, and up/down. The basic result, that there is a broad scattering of response types with no obvious clumping, remains. There is also a slight shift in the distribution toward the bottom edge of the graph.

\section{Spiral tuning}

As described above, most MSTd cells responded to two or even all three types of motion (expansion/contraction, rotation, and

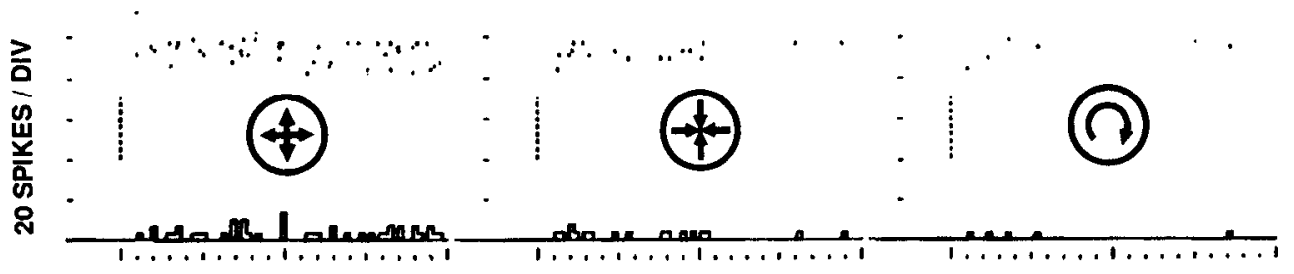

0.1 SEC / DIV
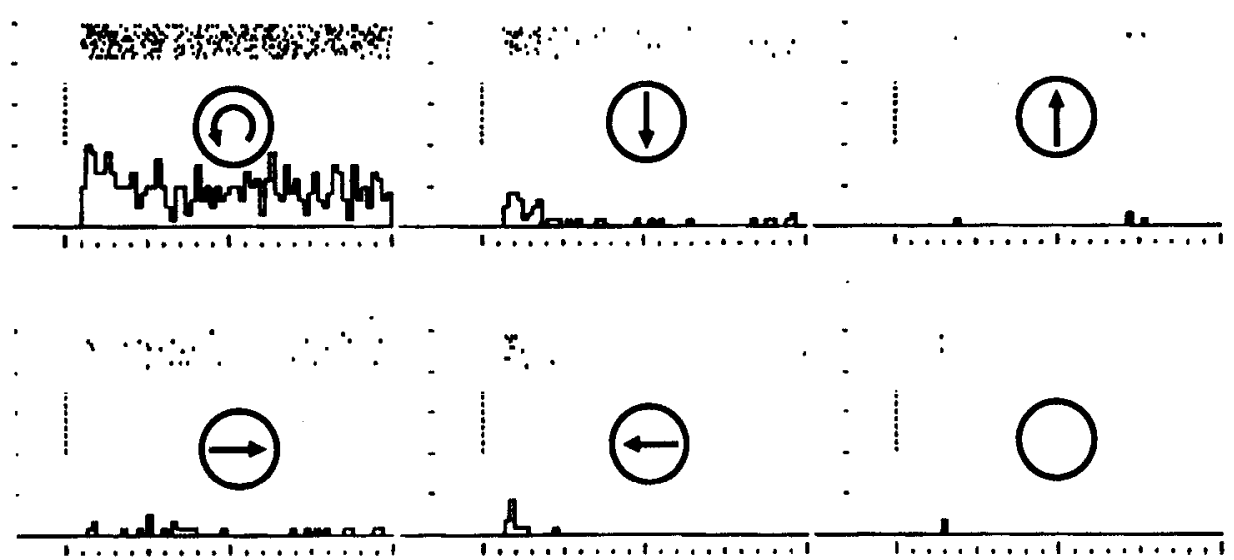

Figure 3. Rasters and histograms for a rotation-selective cell. 
Figure 4. A double-component cell, responding to expansion and to rotation.
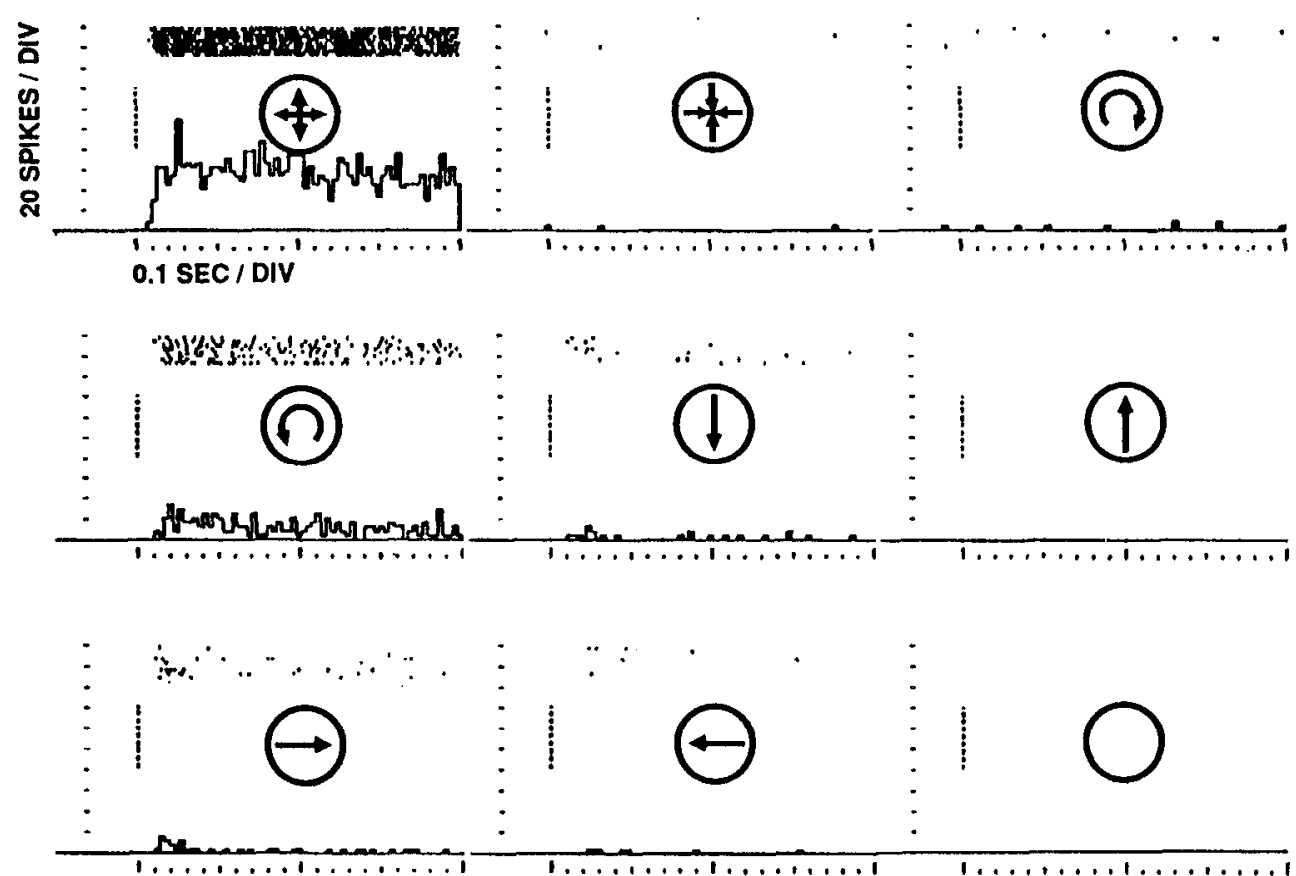

translation). For example, many cells responded to expansion as well as to rotation. We hypothesized that these cells would respond best to an intermediate stimulus, which combined expansion with rotation. To test this hypothesis, we used spiraling stimuli that mixed expanding and rotating motion in various proportions. Whilc the speed was held equal for all stimuli, the pitch of the spiral motion was systematically varied.

Sixty-seven cells were tested with expansion, contraction, both rotations, and four intermediate directions of spiral motion. Sixty-six cells responded significantly to at least one stimulus ( $t$ test, $p<0.05$ ). Figure $7 A$ shows the result for one cell. In this polar plot, the angle represents the pitch of spiral motion, and the radius represents the response of the cell. Thus, eight direc- tions were sampled in this continuous "spiral space." The spontaneous activity for this neuron was near zero. The cell responded to contraction, and also to clockwise rotation, but its preferred stimulus clearly lay between. The cell was tuned to the contracting clockwise stimulus. The response to this preferred spiral stimulus was significantly greater than the response to contraction or to clockwise rotation ( $t$ test, $p<0.05$ ).

Figure $7 B$ shows the same tuning curve, plotted in Cartesian coordinates and fitted with a Gaussian function. The fit is extremely good, with an $r$ value of 0.99 . The location of the peak of the Gaussian gives an estimate of the cell's preferred direction in spiral space. In this case, the preferred direction was near the contracting clockwise spiral, at an angular location of - $47^{\circ}$. The
Figure 5. A multicomponent cell responding to contraction, rotation, and two directions of translation.

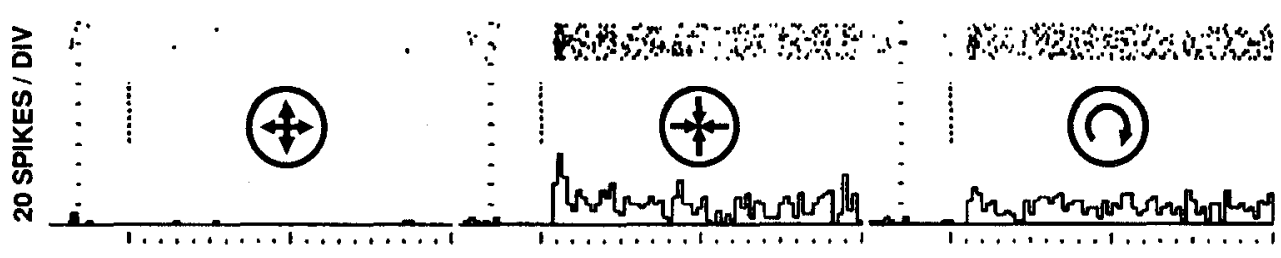

0.1 SEC / DIV
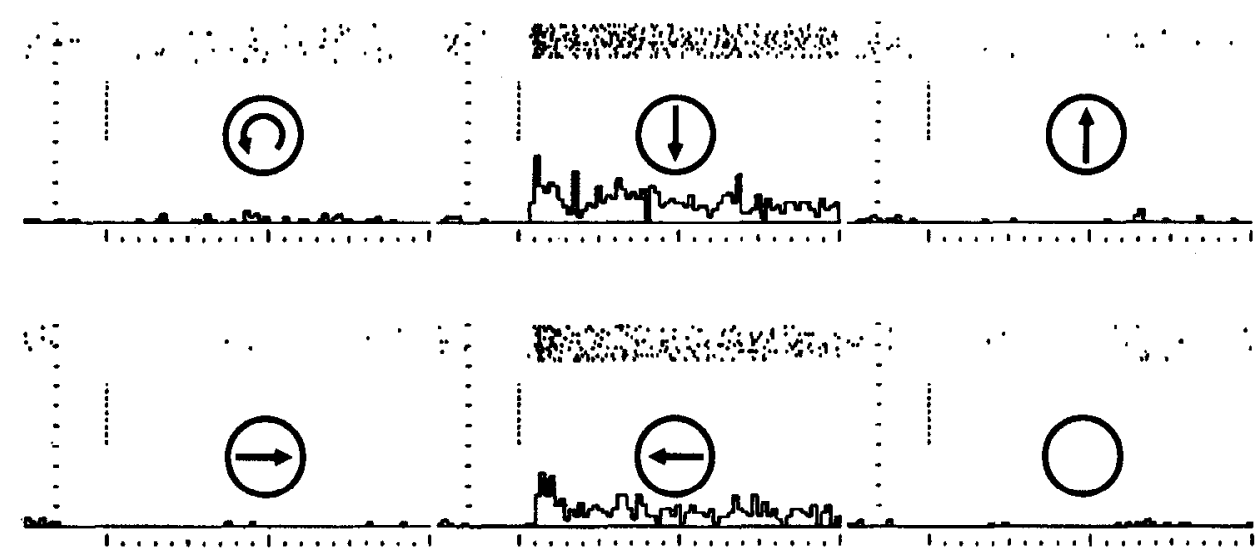
A

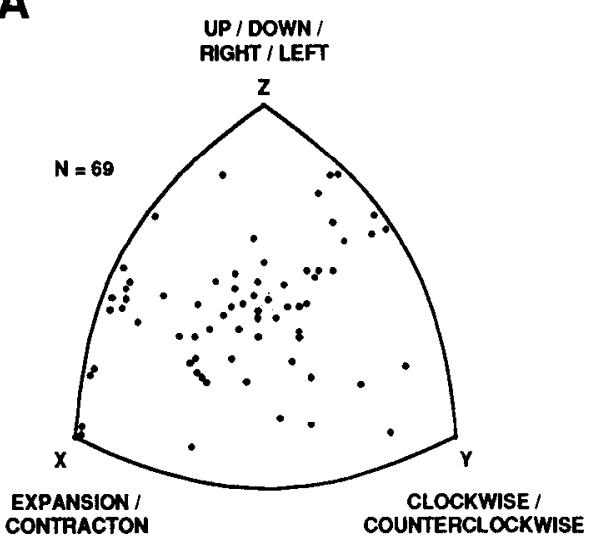

B

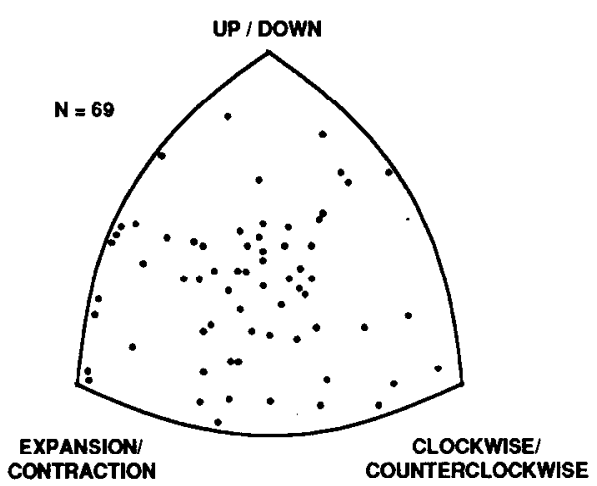

Figure 6. Relative response strength to different types of motion. Each point represents a responsive cell. $A$, A cell plotted close to one vertex responded best to the corresponding type of motion. Cells plotted near the center responded equally well to all three types of motion. The response to each type of motion was calculated in spikes/sec - spontaneous. For a point $P$, the distances $P X, P Y$, and $P Z$ were given by $P X=1 /[K *($ response to $\max ($ expansion, contraction $))+1], P Y=1 /[K *($ response to $\max (\mathrm{clock}$ wise, counterclockwise) $)+1]$, and $P Z=1 /[K \cdot($ response to $\max ($ up,down,right,left)) +1 ], where $K$ is a scaling factor found by numerically solving the three equations for each cell, and max refers to the stimulus of each motion type that elicited the maximum response. Cells that gave inhibitory responses could not be included in this analysis, and therefore only 69 of 75 responsive cells could be plottcd. $B$, Similar to $A$, but comparing expansion/contraction, clockwise/counterclockwise, and up/down.

standard deviation of the Gaussian, $\sigma$, gives an estimate of the width of the cell's tuning curve, and in this case was $45^{\circ}$.

One important question is how much variability there is in the cell's preferred direction. That is, if the cell were tested scveral times, how would the preferred direction change from one test to the next? To answer this question we used the following procedure. We plotted multiple tuning curves for the cell, each based on data from a single trial per stimulus. Since the cell was tested with 10 trials per stimulus, we were able to plot 10 separate tuning curves. Each of these tuning curves was fitted with a Gaussian function, and the location of the peak of the Gaussian was used to estimate the preferred direction. Thus, these calculations gave 10 estimates of the cell's preferred direction. The mean preferred direction was $-47^{\circ}$, shown in Figure $7 A$ as a ray projecting through the tuning curve. The SE was $2.5^{\circ}$. Clearly, this cell was tuned within a very narrow confidence window. That is, the cell responded with such remarkable consistency that repeated trials yielded essentially the same tuning curve.

Figure 8 shows four more examples of tuning curves obtained with the spiral stimuli. Again, each cell is tuned to one preferred stimulus within a narrow confidence window. The cell shown in Figure $8 A$ was tuned to the expanding stimulus. Its preferred direction was $89^{\circ}$, with an SE of $3.5^{\circ}$. The width of its tuning curve, $\sigma$, was $33^{\circ}$, and the $r$ value for the Gaussian fit was 0.99 . The cell in Figure $8 B$ was tuned to the counterclockwise rotation (preferred direction $=175^{\circ}, \mathrm{SE}=6.8^{\circ}, \sigma=39^{\circ}, r=0.99$ ). The cell in Figure $8 \mathrm{C}$ was tuned to the expanding counterclockwise spiral (preferred direction $=133^{\circ}, \mathrm{SE}=11.0^{\circ}, \sigma=57^{\circ}, r=0.97$ ). The cell in Figure $8 D$ was tuned to the expanding clockwise spiral (preferred direction $=59^{\circ}, \mathrm{SE}=3.5^{\circ}, \sigma=38^{\circ}, r=0.99$ ).

The cell shown in Figure $8 D$ is one example of the high degree
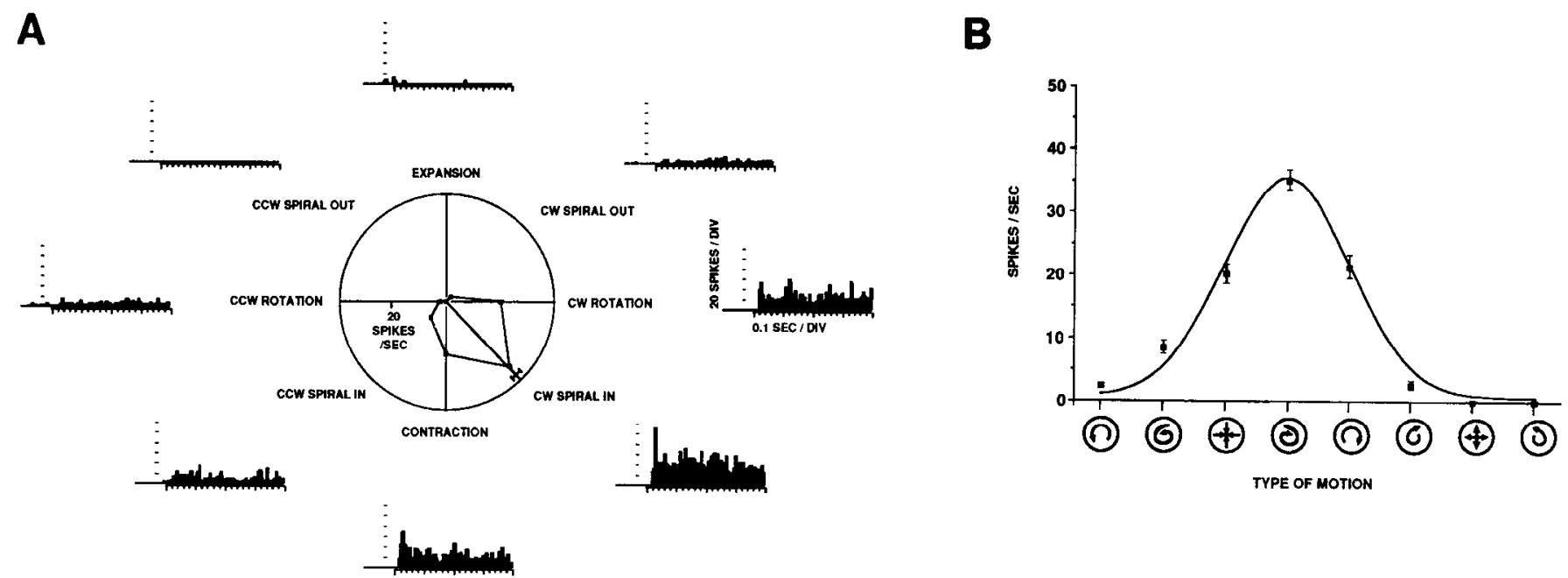

Figure 7. A cell tuned to clockwise contracting spiral. $A$, In this polar plot, the angle represents the type of stimulus and the radius represents the response magnitude. The line directed at $-47^{\circ}$ indicates the cell's best direction as determined by a Gaussian curve fit. The error bar shows an SE of $2.5^{\circ}$ for determining the best direction. The response is also shown in histograms at the perimeter, summed over 10 trials. $C W$, clockwise; $C C W$, counterclockwise. $B$, The tuning curve from $A$ plotted in Cartesian coordinates and fitted with a Gaussian function. The $i c o n s$ on the $\mathrm{x}$-axis indicate the type of stimulus. The error bars show SE across 10 trials. 
A
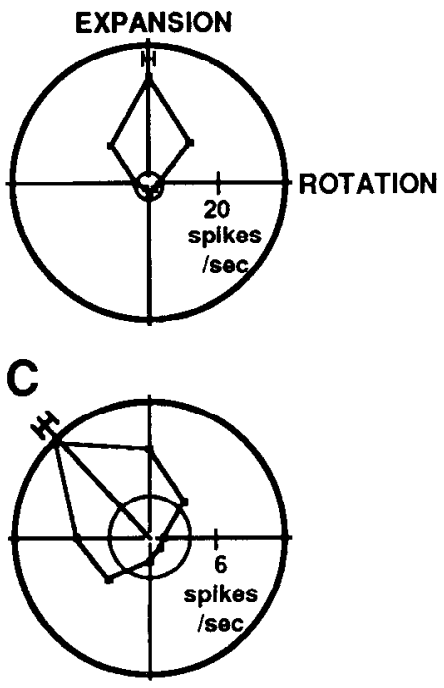

$\mathbf{E}$

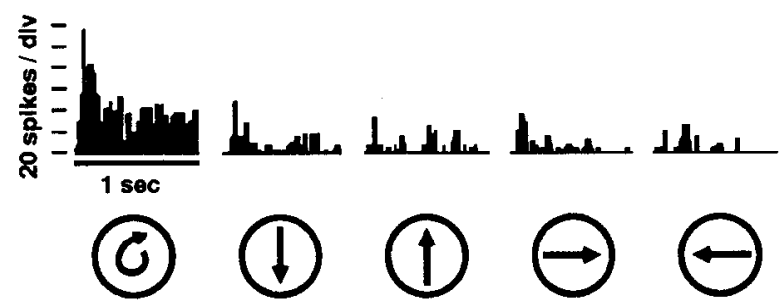

Figure 8. $A-D$, Four examples of tuning curves generated with spiral stimuli (see also Fig. 7). The inner circle shows the spontaneous activity. The ray passing through the tuning curve indicates the preferred direction as calculated with a Gaussian curve fit, and the error bar indicates the $\mathrm{SE}$ in preferred direction. $A$, Expansion-tuned cell. $B$, Rotationtuned cell. $C$ and $D$, Spiral-tuned cells. $E$. Histograms for the cell shown in $D$, demonstrating a large response to the preferred spiral motion and little response to translational motion.

of selectivity among MSTd cells. In addition to spiral motion, this ncuron was tested with translational motion, and the histograms in Figure $8 E$ show that it responded well to spiral and very little to translation. Indeed, the response to the preferred spiral motion was significantly greater than the response to any other stimulus presented ( $t$ test, $p<0.05$ ). In total, 33 of the cells tested with spiral motion were also tested with translational motion. Of these, $24(73 \%)$ responded better to one of the directions in spiral space than to translational motion, while 9 $(27 \%)$ responded better to translation than to any of the directions in spiral space. It is not surprising that some of the cells tuned to expansion, contraction, rotation, or spiral should also respond to translation. The scatter plot in Figure $6 \mathrm{~A}$ demonstrates that most cells respond at least to some extent to translational motion. Perhaps these cells would respond best to an even more complex stimulus combining spiral motion with translation.

While the examples of tuning curves shown in Figures 7 and 8 are compelling, we also analyzed the behavior of the entire sample of cells. For each cell, a tuning curve was plotted and then fitted with a Gaussian function. Fifty-seven cells $(86 \%)$ had smooth tuning curves and good Gaussian fits, with $r>0.9$. Nine cells $(14 \%)$ had more jagged tuning curves, with $r<0.9$. Two of these irregular cells had bidirectional rotation responses, and

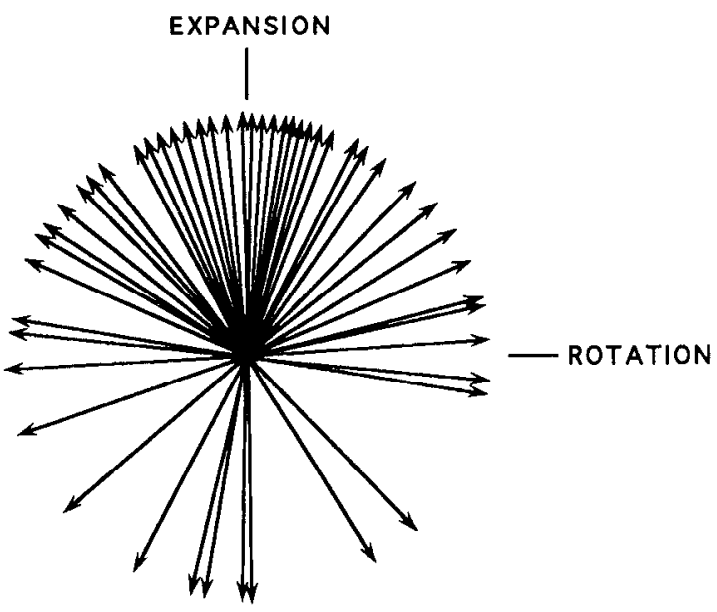

Figure 9. Preferred spiral directions for 57 cells. Each arrow represents a cell. For each cell, the preferred direction was found by fitting the tuning curve with a Gaussian function.

exhibited double-lobed tuning curves. For the 57 single-peak cells, the tuning curves were similar to the examples shown above. For this population of cells, the average $r$ for the Gaussian fit was 0.97 ; the average $\sigma$, or the width of the tuning curve, was $61^{\circ}$; and the average SE, or the confidence limits for the preferred direction, was $5.9^{\circ}$.

For each of the 57 cells, we calculated the direction of best response using the Gaussian fit. Figure 9 shows the result. In this graph, each arrow represents the preferred direction for a single neuron. There is a wide range of best directions, and a strong bias for the expanding stimulus. This bias had been previously reported (Tanaka and Saito, 1989). We categorized the cells as tuned to expansion, contraction, rotation, or spiral, depending on which of these stimuli lay closest to the cell's preferred direction. There were 24 expansion-tuned cells, 20 spiraltuned cells (16 expanding spiral and 4 contracting spiral), 9 rotation-tuned cells, and 4 contraction-tuned cells.

If MSTd decomposed optical flow into an expansion/contraction channel and a separate rotation channel, then most of the cells should be tuned to expansion, contraction, or rotation, and few if any should be tuned to spiral motion. Instead, a large proportion of the cells were tuned to spiral motion, and indeed we encountered more spiral-tuned cells than rotation- or contraction-tuned cells. The most striking pattern to the results was that the expansion stimulus was the most heavily represented, and stimuli progressively farther from expansion were progressively less represented. On this evidence, it appears that MSTd does not use an axis-based method to analyze motion, and instead samples a continuous array of stimuli with a strong bias for the expanding stimulus.

However, one possibility is that the spiral-tuned cells are somehow less selective, or less consistent, and that cells tuned to expansion, contraction, or rotation (termed "cardinal direction-tuned" cells) are more selective and contribute more to the information processing in MSTd. To test this possibility, we compared the spiral-tuned cells to the cardinal direction-tuned cells on the three measures described above: $\sigma, \mathrm{SE}$, and $r$. Spiraltuned cells $(N=20)$ had an average $\sigma$ of $60 \pm 34^{\circ}$, an average $r$ of $0.96 \pm 0.03$, and an average SE of $6.6 \pm 3.8^{\circ}$. Cardinal direction-tuned cells $(N=37)$ had an average $\sigma$ of $62 \pm 25^{\circ}$, an average $r$ of $0.97 \pm 0.02$, and an average SE of $5.6 \pm 4.29^{\circ}$. There was no significant difference between spiral-tuned cells 


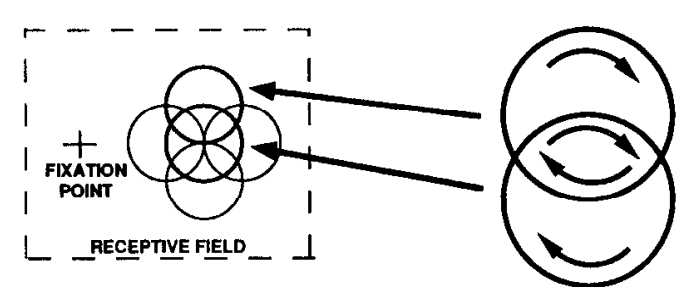

B

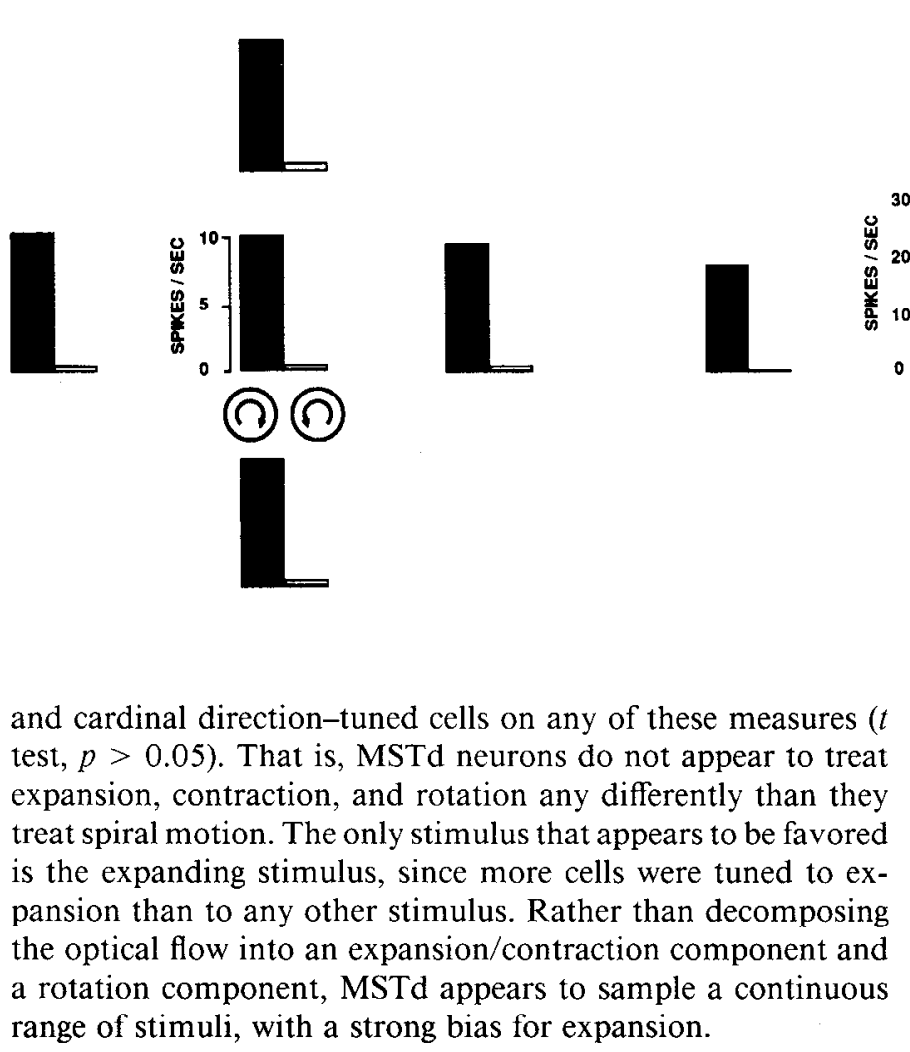

\section{Position invariance}

Responses to expansion, contraction, and rotation were tested with $10^{\circ}$ diameter stimuli at five positions in the receptive field. These positions lay in an overlapping cloverleaf arrangement. As shown in Figure $10 \mathrm{~A}$ for the case of rotation, the local direction of motion can reverse depending on stimulus location. Therefore, if a cell responded in the same way to all five positions, the response could not be explained by local directional selectivity. Cells that pass this test of position invariance must be selective for the global pattern of motion.

Figure $10 B$ shows the result for a cell that preferred clockwise rotation. The cell is position invariant; it responded to clockwise and not to counterclockwise rotation, at all five stimulus positions. Figure $10 C$ shows the result for a cell that preferred expansion. Again, the response is position invariant. Many cells showed a sloping response profile, indicating that the response was stronger at some locations than at others, but in no casc did a cell reverse its selectivity when the stimulus had moved to a different position. The sloping response profile may be due to stimulus positions that were not always optimally centered in the receptive field.
To graph the entire sample of cells, we devised a position invariance index (PI). For each cell, first the directional selectivity (DS) was calculated at each of the five positions using a standard formula [DS $=1-$ (response to antipreferred stimulus/ response to preferred stimulus)]. For example, for an expansion cell, the preferred stimulus was expansion and the antipreferred stimulus was contraction. For a clockwise rotation cell, the preferred stimulus was clockwise rotation, and the antipreferred stimulus was counterclockwise rotation. In this particular test we did not use spiral stimuli. The DS at each surrounding position was then divided by the DS at the central position (PI = $\mathrm{DS}_{\text {surround }} / \mathrm{DS}_{\text {center }}$ ), yielding four numbers per cell. A perfectly position-invariant cell, responding with equal selectivity to all five locations, would have four PI indices all equal to 1 ; a cell that responded more to some locations than to others would have PI values that varied around 1 ; and a cell that reversed its selectivity, responding better to one stimulus in one position and to another stimulus in another position, would have negative PI values.

Figure 11 shows the results for all 52 cells. All of these cells were directionally selective; that is, the response to one stimulus was significantly greater than the response to the opposite stimulus ( $t$ test, $p<0.05$ ). There is a sharp peak centered at $\mathrm{PI}=$ 1 , indicating that most responses were completely position invariant. None of the indices fell below 0 , indicating that no cells reversed their selectivity. The sharpness of the peak is remarkable considering that the PI is calculated with two divisions in sequence, and is therefore particularly sensitive to noise.

Duffy and Wurtz (1991b) have reported that certain of their cell types were more position invariant than others. In particular, single-component cells, which responded to only one of 


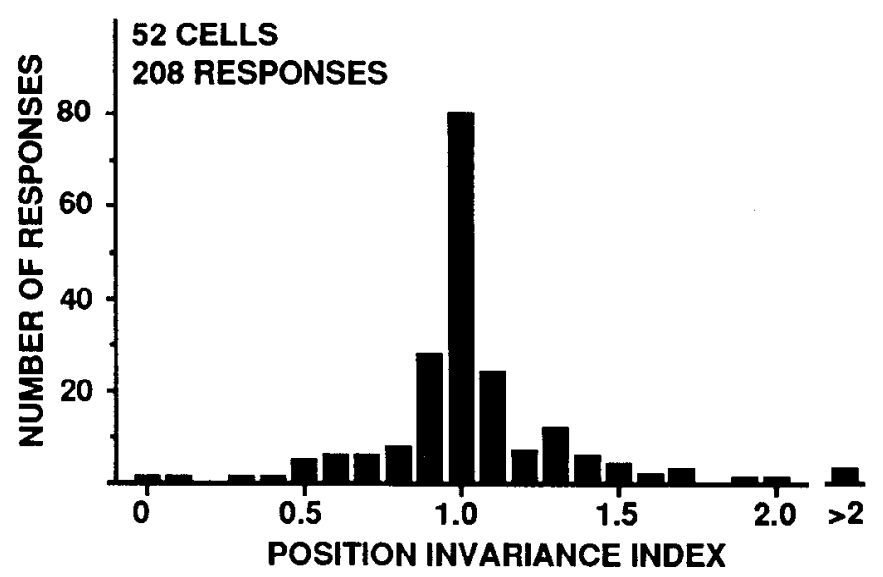

Figure 11. Position invariance index (PI) for a sample of 52 cells (see Results for detailed explanation of PI). PI $=1$ indicates perfect position invariance. Negative values indicate that the cell reversed its stimulus preference depending on the location of the stimulus. The population is sharply peaked around 1 , and none of the responses were negative.

the three classes of motion (translation, rotation, and expansion/ contraction), were more position invariant, and multicomponent cells, which responded to two or all three classes of motion, were less position invariant. We tested our own data for the same trend. We divided cells into two groups, single-component and multicomponent, using the following criteria: if the response to one of the three types of motion was more than twice the response to the other types, the cell was classed as single component; the remainder were classed as multicomponent. This analysis was necessarily limited to 33 cells, which were tested both for their basic stimulus selectivity and for position invariance. Figure 12 shows the result. Single-component cells (Fig. $12 A$ ) were clearly more position invariant, since their graph is more sharply peaked around PI $=1$. Multicomponent cells (Fig. $12 B$ ) were less position invariant, since their graph shows more spread around 1, although they still show a considerable degree of position invariance. Ihis result corroborates Duffy and Wurtz (1991b). However, as described in the next section, the result probably does not reflect a genuinc difference between singleand multicomponent cells; rather, it may be due to a certain kind of sampling error.

\section{Spiral position invariance}

In our test of position invariance described above, as well as in tests by other investigators (Duffy and Wurtz, 1991b), the cells were lested only with rotation, expansion, contraction, and translation. Spiral stimuli were not used. Under these test conditions, cells that are tuned to spiral motion would appear to be "multicomponent," for example, responsive both to rotation and to contraction (such as the neuron shown in Fig. 7A). These spiral-tuned cells would have been tested for position invariance with suboptimal stimuli, that is, the rotations, expansions, and contractions for which they are not ideally tuned. Perhaps their lesser degree of position invariance is simply a result of being tested with nonpreferred stimuli. Indeed, in our formulation, there is no fundamental difference between "multicomponent" and "single-component" cells. We suggest that most cells prefer a single stimulus, but if the preferred stimulus is not included in the experiment, then several nonpreferred stimuli may appear to drive the cell equally well, giving the impression of a multicomponent response. Based on these ideas, we predicted that
A. Single Component Cells

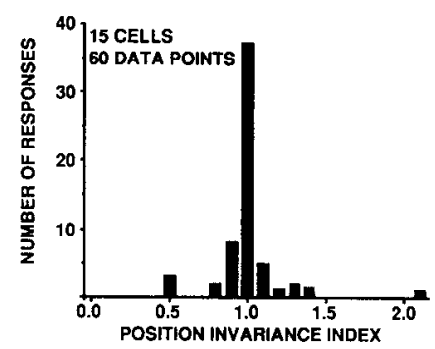

B. Multi Component Cells

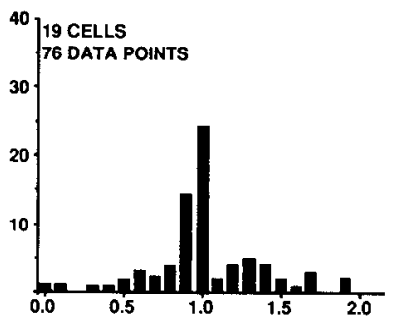

Figure 12. Position invariance for single-component and multicomponent cells. Single-component cells $(A)$ are more position invariant than multicomponent cells $(B)$, since the histogram in $A$ is more sharply peaked around $\mathrm{PI}=1$.

spiral-tuned cells would be just as position invariant as expansion- or rotation-tuned cells, if properly tested with their preferred, spiraling stimulus.

We tested 35 cells by plotting complete spiral tuning curves at two locations in the receptive field. These overlapping locations spanned $25^{\circ}$ of visual angle. Twenty-four cells responded significantly above baseline to both stimulus locations. Figure 13 shows the result for one cell. The cell clearly prefers a spiral stimulus, and prefers the same stimulus at both locations. In addition, the shape of the tuning curve is the same at both locations. The preferred direction for the upper position, determined with a Gaussian curve fit, is $115^{\circ}$, corresponding to an expanding counterclockwise spiral. The preferred direction for the lower position is $116^{\circ}$. This robust position invariance indicates that the cell is indeed selective for spiral motion.

Figure $14 \mathrm{~A}$ shows another example of a cell tuned to spiral motion. $\Lambda$ gain, the cell prefers the same stimulus at both locations. However, the response to expansion, which is on the flank of the tuning curve, varies from one location to the other. The result from this particular cell supports our hypothesis. Tested only with expansion and rotation, this cell would appear to be multicomponent, and somewhat position dependent. However, by testing the cell with its preferred spiraling stimulus, we have demonstrated a much higher degree of position invariance.

Figure $14 B$ shows a cell tuned to expansion. Once again, the cell prefers the same stimulus at both retinal locations. In this case, however, even an expansion-tuned cell appears variable when tested with a stimulus on the flank of the tuning curve. The expanding clockwise spiral gives a good response in the lower position, but very little response in the upper position. These results demonstrate how spiral-tuned cells and expansiontuned cells are not different. Both types are position invariant when tested with their preferred stimulus, and somewhat position dependent when tested with a nonpreferred stimulus.

For each cell, the upper and lower position tuning curves were fitted with Gaussian functions. Two of the 24 cells with significant responses had irregular tuning curves with poor Gaussian curve fits $(r<0.9)$, and were eliminated from the analysis. For the remaining 22 cells, the preferred direction was calculated from the Gaussian curve fit, and the cells were categorized as tuned to expansion, contraction, rntation, or spiral, depending on which of these stimulus directions was closest to the cell's preferred direction. Eight cells were tuned to spiral motion, and 14 were tuned to expansion, contraction, or rotation ("cardinal direction-tuned" cells). 


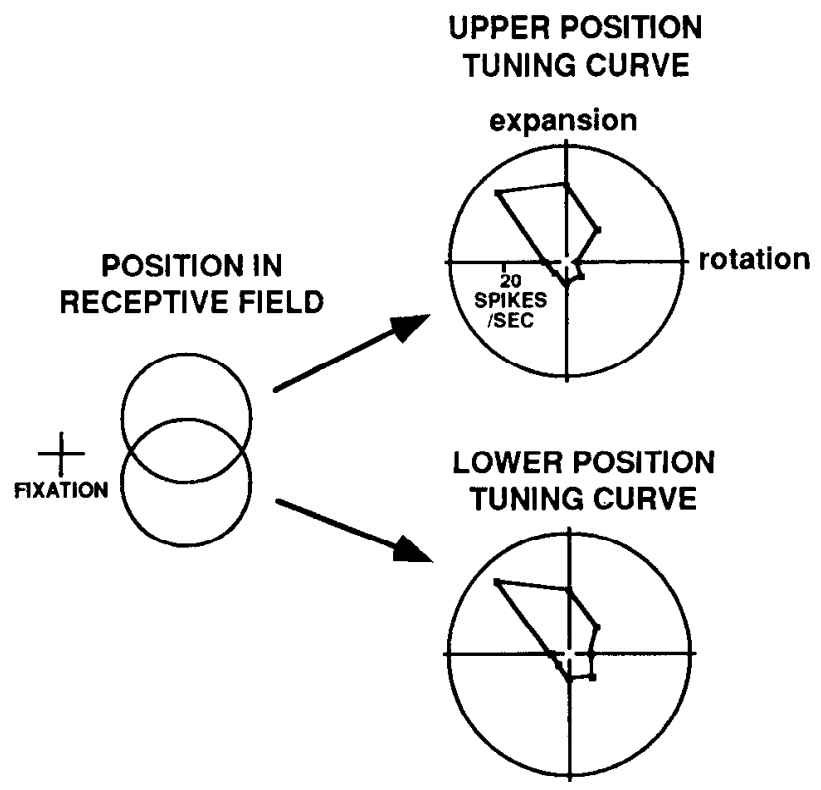

Figure 13. Position invariance for a spiral-selective cell. Full spiral tuning curves were plotted at two overlapping locations spanning $25^{\circ}$ vertically.

A simple measure of position dependence is the shift in preferred direction from one stimulus location to the other. A random distribution would give an average shift of $90^{\circ}$. Our sample of 22 cells gave an average shift of $10.7^{\circ}$. This result adds to the previous result (see Position invariance, above), that MSTd responses remain impressively similar when the stimulus is moved to a different location. The average shift for spiral-tuned cells $\left(11.2 \pm 8.8^{\circ}\right)$ and the average shift for cardinal direction-tuned cells $\left(10.5 \pm 6.5^{\circ}\right)$ were not significantly different $(t$ test, $p>$ 0.05 ), showing that the two groups of cells were equally position invariant.

For further analysis we devised a second measure of position dependence: the change in response, or $\Delta R$. First the directional selectivity (DS) was calculated for each pair of stimuli (e.g., expansion vs contraction) using a standard formula [DS $=1-$ (response to antipreferred stimulus/response to preferred stimulus)]. We calculated the DS for both the upper and lower stimulus positions, and then subtracted the two measures to arrive at $\Delta R\left(\Delta R=\left|\mathrm{DS}_{\text {uppor }}-\mathrm{DS}_{\text {lower }}\right|\right)$. A $\Delta R$ of 0 indicates that the cell responded with the same selectivity to both stimulus locations, and was therefore position invariant. Larger values of $\Delta R$ indicate larger discrepancies between the two responses, and therefore greater amounts of position dependence.

For each cell, $\Delta R$ was calculated for the cell's preferred direction $\left(\Delta K_{\text {preferred }}\right)$, the direction $45^{\circ}$ off from preferred $\left(\Delta R_{45}\right)$, and the direction $-45^{\circ}$ off from preferred $\left(\Delta R_{-45}\right)$. For example, for a cell that preferred an expanding clockwise spiral, $\Delta R_{\text {preferred }}$ was taken along the expanding clockwise direction, $\Delta R_{45}$ was taken along the expanding direction, and $\Delta R_{-45}$ was taken along the clockwise rotating direction. We predicted that $\Delta R_{\text {preferred }}$ would be smaller than $\Delta R_{45}$ and $\Delta R_{-45}$, indicating greater position invariance to the preferred stimulus, and less position invariance to the two flanking stimuli. Table 1 shows the results, in agreement with prediction. The mean of $\Delta R_{\text {prefered }}$ was 0.08 , the mean of $\Delta R_{45}$ was 0.17 , and the mean of $\Delta R_{-45}$ was 0.23 . This effect was statistically significant $(2 \times 3$ ANOVA for mixed design, unequal $N ; F=3.50, p<0.05$ ). That is, cells were significantly less position dependent (i.e., more position in-

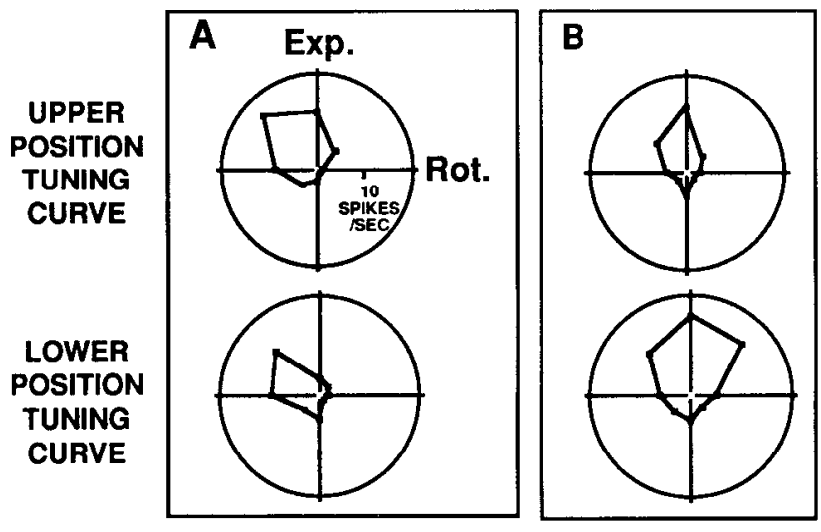

Figure 14. Two examples of cells tested at two retinal locations. A, A spiral-tuned cell. $B$, An expansion-tuned cell. See also Figure 13.

variant) when tested with their preferred stimulus than when tested with nonpreferred stimuli. There was no significant difference between spiral-tuned cells and cardinal direction-tuned cells $(F=1.33, p>0.05)$, and there was also no significant interaction between the two variables $(F=1.25, p>0.05)$.

These results support the view that spiral-tuned cells are not fundamentally different from cardinal direction-tuned cells. That is, when tested with their preferred stimulus, both types of cells are equally position invariant. Also, both types of cells are equally position dependent when tested with stimuli that are offset from the preferred direction, such as an expansion stimulus in the case of a spiral-tuned cell, or a spiral stimulus in the case of an expansion-tuned cell.

\section{Size}

We tested 20 cells for the effect of stimulus size on response. Each cell was tested with its preferred stimulus at two different sizes: $10^{\circ}$ and $20^{\circ}$. Cells typically responded to both stimuli but less to the smaller stimulus. As shown in Figure 15, on average the response to the $10^{\circ}$ stimulus was $76 \%$ as strong as the response to the $20^{\circ}$ stimulus.

\section{Discussion}

\section{Decomposition hypothesis}

All complex motion patterns can be decomposed into expansion, rotation, and translation. Therefore, a potentially powerful way for MSTd to encode optical flow would be to use separate

Table 1. Position dependence of spiral-tuned and expansion-, contraction-, and rotation-tuned neurons

\begin{tabular}{llll} 
& \multicolumn{2}{l}{ Factor $\mathrm{B}(\Delta R)$} \\
\cline { 2 - 4 } Factor A (cell type) & $\Delta R_{\text {preferred }}$ & $\Delta R_{45}$ & $\Delta R_{-45}$ \\
\hline $\begin{array}{l}\text { Spiral-tuned cells } \\
\quad(N=8)\end{array}$ & 0.06 & 0.25 & 0.27 \\
$\begin{array}{l}\text { Exp., Con., and Rot. tuned cells } \\
\quad(N=14)\end{array}$ & 0.09 & 0.12 & 0.21 \\
$\quad \begin{array}{l}\text { Total mean } \\
(N=22)\end{array}$ & 0.08 & 0.17 & 0.23
\end{tabular}

Data are mean $\Delta R$ for preferred and nonpreferred stimuli. Cells were tested with spiral stimuli at two overlapping retinal locations (see Fig. 13). A measure of position dependence was calculated for the preferred spiral direction $\left(\Delta R_{\text {preferred }}\right)$, the direction $45^{\circ}$ off from preferred $\left(\Delta R_{45}\right)$, and the direction $-45^{\circ}$ off from preferred $\left(\Delta R_{-45}\right)$. Spiral-tuned cells were compared to cardinal direction-tuned cells on these three measures of position dependence. 


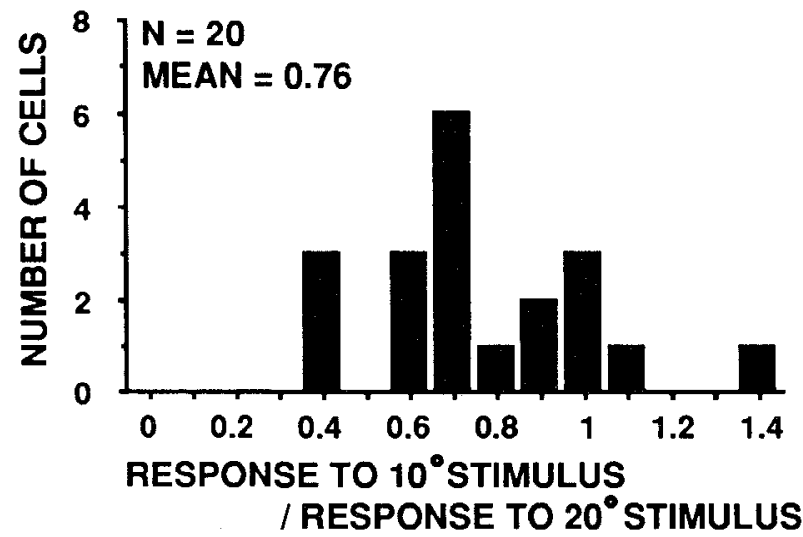

Figure 15. Twenty cells tested with stimuli of two different sizes. On average, the response to the $10^{\circ}$ stimulus was $76 \%$ the response to the $20^{\circ}$ stimulus.

neuronal channels for these three motion components. In this model, only the cells that respond purely to one type of motion contribute to the final stages of analysis. Cells that respond to more than one type of motion might lie lower down in the information processing hierarchy, or might be used for some other type of motion task. Past results demonstrating that singlecomponent cells are more position invariant and generally more reliable (Duffy and Wurtz, 1991a,b) are consistent with this conception of MSTd.

We addressed this decomposition issue by concentrating on responses to expansion and rotation. Expansions and rotations span a two-dimensional stimulus space, which we have plotted with expansion on the vertical axis and rotation on the horizontal axis. Directions between the cardinal axes represent spiral motion pitched to various degrees. We tested cells with eight different directions in this spiral space, to determine if MSTd decomposes optical flow into an expansion channel and a rotation channel or if some cells prefer intermediate stimuli. The results showed a range of preferred stimuli. Most cells showed smooth and clearly oriented tuning curves, which were well approximated by Gaussian functions. In particular, doublecomponent cells, responding to expansion and to rotation, turned out to have a single preferred stimulus, a spiraling stimulus that lay between expansion and rotation. This response to the spiral stimulus was often much greater than the response to expansion, rotation, or translational motion. Thus, there do not appear to be separate channels for expansion and rotation in MSTd.

However, several alternative explanations must be considered. First, can speed tuning confound the preferred direction of the cell? For example, a cell might prefer a stimulus that expands at a particular rate. If our standard expansion stimulus happens to be faster than the cell's preferred stimulus, then the cell would not respond at its maximum rate. The spiral stimulus, however, contains an expansion component that is about $70 \%$ as large as in the pure expansion stimulus. Therefore, the cell could respond better to the spiral stimulus, not becausc it was tuned to spiral motion, but because it was tuned to expansion of a particular speed. However, this explanation cannot account for any of our results, for in this case the cell would respond equally well to an expanding clockwise spiral and to an expanding counterclockwise spiral. We would obtain a tuning curve with two peaks offset by $90^{\circ}$, yet we never encountered a single cell with this kind of tuning curve. Indeed, the spiral-tuned cells that we studied responded best to only one spiral stimulus.
Another question is whether the space for spiral tuning is truly continuous, or if there are only eight channels including four for spiral stimuli. It appears that the space is continuous, since we found many cells with tuning curves for which the peak of the Gaussian curve fit lay between the sample points (as shown in Fig. 9). Recently, we have tested cells with 16 directions, and found tuning curves with peaks between the eight directions tested in the present study (B. Geesaman and R. A. Andersen, unpublished observations). This finding lends further support to the proposal that MSTd cells continuously sample the spiral space.

It is clear why cells tuned to spirals might have developed. Every time a rotating object approaches the observer, the combination of rotation and expansion will produce a spiral. Also, cvery time the observer moves forward and simultaneously tracks a point to the side, the optical flow field will appear to spiral around that fixation point. Indeed, pure rotation and pure expansion are limiting cases, and must occur with far less frequency than spiral motion. However, it seems that expansion is treated in a special fashion in MSTd, since the distribution of spiral responses is strongly biased toward the expanding stimulus. This bias may reflect the special importance of objects approaching the observer, or the typical forward motion of the observer through the environment.

We suggest that most MSTd cells are tuned to a single preferred stimulus. This idea is an important simplification. The multicomponent responses described in previous studies (Duffy and Wurtz, 1991a) may be indicative of a continuous tuning over many different stimulus patterns. A cell that appears to be multicomponent may be tuned to an intermediate stimulus that was not included in the experiment. The recent experiments of Duffy and Wurtz (1991c) also support this idea, since they find that double-component, translation and expansion cells will give even stronger responses to a combination of the two stimuli. Taken together, these data suggest that most MSTd cells are really single component, in that they respond best to one preferred stimulus.

\section{Position invariance}

We found that responses to expansion, contraction, rotation, and spiral were mostly unaffected by moving the stimulus to different locations within the receptive field. Although the strength of the response varied somewhat with position, the preferred direction in spiral space never changed.

Position invariance is remarkable if one considers the complexity of the mechanisms needed to achieve it. The simplest model is a hierarchy, where various position-dependent cells converge on a higher-order cell to create position invariance. However, this model seems unlikely because we found no position-dependent cells. Instead, there must be a highly complicated convergence from the local, direction-selective responses of MT to the fully position invariant, motion-pattern-selective cells of MSTd. Although several interesting models exist to show how position invariance might be achieved (Poggio et al., 1991a,b; Sereno and Sereno, 1991; Lappe and Rauschecker, 1993; Zhang et al., 1993), the present data say very little about how this convergence is achieved.

\section{Spiral position invariance}

It has been reported that some MSTd cells are more position invariant than others. In particular, single-component cells (responsive to only one type of motion) are more position invari- 
ant, and multicomponent cells (responsive to two or three motion types) are less position invariant (Duffy and Wurtz, 1991b). Our own data showed the same trend.

In our model, we suggest that most cells prefer a single stimulus, but if the preferred stimulus is not included in the experiment, then several nonpreferred stimuli may appear to drive the cell equally well, giving rise to the multicomponent response. We hypothesized that multicomponent cells appear to be less consistent only because they are being tested with nonpreferred stimuli. Our finding that the flanks of the tuning curves in spiral space are less position invariant than the center is consistent with this interpretation. For instance, a double-component cell, responding to rotation and to expansion, will show poor position invariance when tested with rotation and expansion stimuli. However, when it is tested with its most preferred stimulus, the intermediate spiral motion, then its position invariance is quite good.

\section{Extent of receptive field in test of position invariance}

Previous studies of MSTd have tested position invariance across a large portion of the receptive field. In one study (Duffy and Wurtz, $1991 \mathrm{~b}$ ), a full $100^{\circ}$ of visual angle was tested. Our own tests covered a smaller extent. In our first test of position invariance, the stimuli were shifted across $20^{\circ}$ of visual angle, and in the test of spiral position invariance, the stimuli were shifted across $25^{\circ}$. One reason for testing a smaller portion of the receptive field is that when stimuli are moved to distal locations, they might fall partly outside the receptive field, or in a weakly responding region at the edge. While testing for position invariance, we sometimes found that a cell responded poorly to one stimulus location, and in each case we found that we had been testing near the edge of the receptive field. In these cases we moved the stimuli farther into the receptive field and recommenced the test. The most important issue, however, is whether our results, gathered over $25^{\circ}$ of visual angle, match the results from previous experiments that have tested over greater extents of the receptive field. Clearly the results match. We have found, like previous researchers (Duffy and Wurtz, 1991b), that most cells show a considerable degree of position invariance. Finally, other data from our lab (Geesaman and Andersen, unpublished observations) show that MSTd cells tuned to spiral motion remain tuned to the same spiral even when the stimulus centers are moved by $40^{\circ}$.

\section{Optical flow and navigation}

When a monkey runs through the forest, he may determine his direction of self-motion by analyzing the resulting optical flow. This optical flow contains a mixture of expansion, produced by the forward motion of the animal, and translation and rotation, produced by eye and head movements. One method of solving this problem of navigation is to decompose the flow field, extracting the expansion component and discarding the rest, and to take the locus of expansion as the direction of self-motion (Gibson, 1950; Longuet-Higgins and Prazdny, 1980; Regan and Beverly, 1982). Human subjects can find the direction of selfmotion to a high degrec of accuracy, even in cases complicated by eye movements, indicating that they are successfully extracting the expansion component (Warren and Hannon, 1988).

Since there are cells in MSTd sensitive to expanding stimuli, it has been suggested that MSTd contributes to visual navigation (Saito et al., 1986; Roy and Wurtz, 1990). It is natural to ask, as we did in our experiment, whether MSTd neurons might be extracting the expansion component in order to determine the direction of heading. We tested this hypothesis by using expansion, contraction, and intermediate spiral stimuli. If MSTd neurons decompose optical flow into an expansion component and a rotation component, then most neurons should be tuned either to expansion or to rotation, but not to the intermediate spirals. However, we found a continuous range of preferred directions, including many cells that were tuned to the intermediate spirals. Therefore, it does not appear that MSTd extracts the expansion component from other components of optical flow. This question of component decomposition was addressed in a different way by Orban et al. (1992), following our preliminary work on spiral selectivity (Graziano et al., 1990). Orban et al. tested expansion-tuned cells with two stimuli: one contained pure expansion, and the other contained the same amount of expansion but had a rotational component added. Despite the fact that both stimuli contained the same degree of expansion, the cells responded negligibly to the spiral stimuli. That is, the cells did not extract the amount of expansion present in the stimulus, and this result is further support for the idea that area MST neurons do not perform a decomposition. Another important conclusion we can draw, in light of the Orban et al. experiment, is that the spiral tuning we found is not a result of a linear addition of expansion and rotation components. If such a linear summation of components existed for MST neurons, then adding rotation to the expansion component should not have reduced the response to the expansion component.

A second difficulty with the navigation hypothesis is that cells contributing to navigation should locate the center of expansion, thereby indicating the animal's direction of motion. The position-invariant responses described in the present article cannot encode the center of expansion in any straightforward way. It is still possible that MSTd uses a coarse coding method, where each cell is fairly position independent but taken together the cells can pinpoint the center of expansion. However, any simple formulation of the navigation hypothesis must be rejected.

A third difficulty with the navigation hypothesis is that flow field cells should be primarily sensitive to stimuli that encompass a large portion of the visual field, while the present experiments demonstrate good responses to stimuli as small as $10^{\circ}$ across. Tanaka and Saito (1989) reported that MSTd cells respond primarily to stimuli $40^{\circ}$ or larger, but these experiments were done in anesthetized monkeys, and the anesthetic may have weakened the response until only very large and salient stimuli could reliably drive the cells. Indeed, in our experiment, the animal was seated in a lighted room, fixating an LED mounted in a stationary visual scene. Only the $10^{\circ}$ stimulus moved. This visual environment does not resemble the flow fields produced by the forward motion of the observer.

Perhaps one function of MSTd is to analyze the motion of individual objects within the environment, an idea originally put forth by Sakata et al. $(1985,1986)$. Any rigid textured object that turns or moves in depth will produce expansion, rotation, spiraling motion, or translation. A region of the brain designed to analyze these types of motion would be useful in parsing the visual world into separate objects and surfaces moving in complicated ways. For example, when a monkey turns an object in his hand, the same types of motion are generated as drive cells in MSTd. In a more general formulation, MSTd might process all kinds of visual motion, regardless of whether the information will be used for visual navigation or for analyzing the motion of objects and surfaces. Our "motion pattern" hypothesis, then, 
states that MSTd neurons are selective for complex patterns of motion per se, independently of whether the motions are generated by self-motion or object motion.

\section{Analogy to IT}

We suggest an analogy between MSTd and inferotemporal (IT) cortex. Both areas appear to code complex patterns, static properties of shape, texture, and color for IT (Gross et al., 1972; Desimone et al., 1984), and kinetic properties for MSTd. Both areas demonstratc position invariance (for IT, Schwartz et al., 1983; Desimone et al., 1984). Position invariance is useful for object analysis, whether for object form or object motion, because an object can appear at many different retinal locations but must maintain perceptual constancy. Both areas have large bilateral receptive fields, and yet emphasize parafoveal retina. Indeed, MSTd appears to contain primarily a central field representation (Desimone and Ungerleider, 1986). This emphasis on central vision is probably also important for object analysis, since the attended object would most likely be foveated. Finally, IT does not use any obvious set of basis vectors to encode shape (Albright et al., 1985), and instead appears to use a complicated population code in which cells are tuned to a wide range of shapes. The present experiments suggest that MSTd also does not use basis vectors and, like IT, uses a population code in which cells are tuned to a wide range of motion stimuli.

It appears that V1, MT, and MSTd are arranged in a motion processing hierarchy (e.g., Maunsell and Newsome, 1987; Andersen, 1989; Boussaoud et al., 1990), where the responses in MT, still relatively simple and local, are combined into the massively complex responses of MSTd. This motion pathway may continue farther to area STP, where rotation- and expansion-selective cells have also been found (Bruce et al., 1981; Hikosaka et al., 1988). Similarly, a simplified hierarchy of object recognition areas has been traced, from $\mathrm{V} 1$, to $\mathrm{V} 2$, to $\mathrm{V} 4$, and then to IT (e.g., Ungerleider and Mishkin, 1982; Desimone et al., 1985). Again, the relatively simple and local properties of V4 cells are combined into the massively complex properties of IT cells. This leap from V4 to IT has been difficult to explain. If MSTd and IT share the same mechanisms, then perhaps studying MSTd will provide clues for comprehending this transformation.

\section{References}

Albright TD (1984) Direction and orientation selectivity in visual area MT of the macaque. J Neurophysiol 52:1106-1130.

Albright TD, Charles R, Gross CG (1985) Inferior temporal neurons do not seem to code shape by the method of Fourier descriptors. Soc Neurosci Abstr 11:1013.

Andersen RA (1989) Visual and eye movement functions of the posterior parietal cortex. Annu Rev Neurosci 12:337-403.

Andersen RA, Graziano MSA, Snowden R (1990) Translational invariance and attentional modulation of MST cells. Soc Neurosci Abstr $16: 7$.

Boussaoud D, Ungerleider LG, Desimone R (1990) Pathways for motion analysis: cortical connections of the medial superior temporal and fundus of the superior temporal visual areas of the macaque. $J$ Comp Neurol 296:462-495.

Bruce C, Desimone R, Gross C (1981) Visual properties of neurons in a polysensory area in superior temporal sulcus of the macaque. $\mathrm{J}$ Neurophysiol 46:369-384.

Desimone R, Ungerleider LG (1986) Multiple visual areas in the superior temporal sulcus of the macaque. J Comp Neurol 248:164189.

Desimone R, Albright TD, Gross CG, Bruce CJ (1984) Stimulus selective properties of inferior temporal neurons in the macaque. $\mathrm{J}$ Neurosci 4:2051-2062.
Desimone R, Schein SJ, Moran J, Ungerleider LG (1985) Contour, color, and shape analysis beyond the striate cortex. Vision Res 25: $441-452$.

Duffy CJ, Wurtz RH (199la) Sensitivity of MST neurons to optic flow stimuli. I. A continuum of response selectivity to large field stimuli. J Neurophysiol 65:1329-1345.

Duffy CJ, Wurtz RH (1991b) Sensitivity of MST neurons to optic flow stimuli. II. Mechanisms of response selectivity revealed by small field stimuli. J Neurophysiol 65:1346-1359.

Duffy CJ, Wurtz RH (1991c) MSTd neuronal sensitivity to heading of motion in optic flow fields. Soc Neurosci Abstr 17:441.

Gallyas F (1979) Silver staining of myelin by means of physical de velopment. Neurol Res 1:203-209.

Geesaman B, Andersen RA (1992) Area MST represents abstract features of motion for a broad range of stimuli. Soc Neurosci Abstr 18 : 1034.

Gibson JJ (1950) The perception of the visual world. Boston: Houghton Mifflin.

Graziano MSA, Andersen RA, Snowden R (1990) Stimulus selectivity of neurons in macaque MST. Soc Neurosci Abstr 16:7.

Gross CG, Rocha-Miranda CE, Bender DB (1972) Visual properties of neurons in inferotemporal cortex of the macaque. J Physiol (Lond) 35:96-111

Heeger DJ, Jepson A (1990) Visual perception of three-dimensional motion. Neural Comp 2:129-137.

Hikosaka K, Iwai E, Saito H, Tanaka K (1988) Polysensory properties of neurons in the anterior bank of the caudal superior temporal sulcus of the macaque monkey. J Neurophysiol 60:1615-1637.

Hubel DB, Wiesel TN (1962) Receptive fields, binocular interaction and functional architecture in the cat's visual cortex. J Physiol (Lond) 160:106-154.

Koenderink JJ, van Doorn AJ (1981) Exterospecific component of the motion paralax field. J Opt Soc Am 71:953-957.

Komatsu H, Wurtz RH (1988a) Relation of cortical areas MT and MST to pursuit eye movements. I. Localization and visual properties of neurons. J Neurophysiol 60:580-603.

Komatsu H, Wurt7. RH (1988h) Relation of cortical areas MT and MST to pursuit eye movements. III. Interaction with full-field visual stimulation. J Neurophysiol 60:621-644.

Lappe M, Rauschecker JP (1993) A neural network for the processing of optic flow from ego-motion in man and higher mammals. Neural Comput 5:374-391.

Livingstone M, Hubel D (1988) Segregation of form, color, movement, and depth: anatomy, physiology, and perception. Science 240:740749.

Longuet-Higgins HC, Prazdny K (1980) The interpretation of a moving retinal image. Proc R Soc Lond [Biol] 208:385-397.

Maunsell JHR, Newsome WT (1987) Visual processing in monkey extrastriate cortex. Annu Rev Neurosci 10:363-401.

Maunsell JHR, Van Essen DC (1983a) The connections of the middle temporal visual area (MT) and their relationship to a cortical hierarchy in the macaque monkey. J Neurosci 3:2563-2580.

Maunsell JHR, Van Essen DC (1983b) Functional properties of neurons in middle temporal visual area of the macaque monkey. I: Selectivity for stimulus direction, speed, and orientation. J Neurophysiol 49:1127-1147.

Orban GA, Lagae L, Verri A, Raiguel S, Xiao D, Maes H, Torre V (1992) First order analysis of optical flow in monkey brain. Proc Nat Acad Sci USA 89:2595-2599.

Poggio I', Verri A Torre V (1991a) Green theorems and qualitative properties of the optical flow. MIT A.I. Memo 1289.

Poggio T, Verri A, Torre V (1991b) Does cortical area MST know green theorems? IRST Technical Report 9008-07.

Regan D, Bever'ey K (1982) How do we avoid confounding the direction we are looking and the direction we are moving? Science 215: 194-196.

Rieger J, Lawton DT (1985) Processing differential image motion. J Opt Soc Am 2:354-359.

Roy JP, Wurtz RH (1990) The role of disparity-sensitive cortical neurons in signalling the direction of motion. Nature 348:160-162.

Saito HA, Yukie M, Tanaka K, Hikosaka K, Fukada Y, Iwai E (1986) Integration of direction signals of image motion in the superior temporal sulcus of the macaque monkey. J Neurosci 6:145-157.

Sakata H, Shibutani H, Kawano K, Harrington TL (1985) Neural mechanisms of space vision in the parietal association cortex of the monkey. Vision Res 25:453-463. 
Sakala H, Shibutani H, Ito Y, Tsurugai K (1986) Parietal cortical neurons responding to rotary movement of visual stimulus in space. Exp Brain Res 61:658-663.

Schwartz EL, Desimone R, Albright I, Gross C (1983) Shape recognition and inferior parietal neurons. Proc Natl Acad Sci USA 80: 5776-5778.

Sereno MI, Sereno ME (1991) Learning to see rotation and dilation with a Hebb rule. In: Advances in neural information processing systems 3 (Lippmann RP, Moody J, Touretzky DS, eds), pp 320326. San Mateo, CA: Morgan Kaufman.

Tanaka K, Saito H (1989) Analysis of motion of the visual field by direction, expansion/contraction, and rotation cells clustered in the dorsal part of the medial superior temporal area of the macaque monkey. J Neurophysiol 62:626-641.

Tanaka K, Hikusaka K, Saito H, Yukie M, Fukada Y, Iwai E (1986) Analysis of local and wide-field movements in the superior temporal visual areas of the macaque monkey. J Neurosci 6:134-144.
Tanaka K, Fukada Y, Saito H (1989) Underlying mechanisms of the response specificity of expansion/contraction and rotation cells clustered in the dorsal part of the medial superior temporal area of the macaque monkey. J Neurophysiol 62:642-656.

Ungerleider LG, Desimone R (1986a) Projections to the superior temporal sulcus from the central and peripheral field representations of V1 and V2. J Comp Neurol 248:147-163.

Ungerleider LG, Desimone R (1986b) Cortical connections of visual area MT in the macaque. J Comp Neurol 248:190-222.

Ungerleider LG, Mishkin M (1982) Two cortical visual systems. In: Analysis of visual behaviour (Ingle DJ, Goodale MA, Mansfield RJN, eds), pp 549-586. Cambridge, M $\Lambda$ : MIT Press.

Warren WH, Hannon DJ (1988) Direction of self motion is perceived from optical flow. Nature 336:162-163.

Zhang K, Sereno MI, Sereno ME (1993) Emergence of position-independent detectors of sense of rotation and dilation with Hebbian learning: an analysis. Neural Comput, in press. 